\title{
What should Autoimmune Thyroiditis be considered for?
}

\author{
Shoichi Kikuchi, Fumio Matsuzuka, Tsukasa Aihara, Chiaki Yasui, Hidenori Yanagi, Masao Mitsunobu
}

Naoki Yamanaka, Ayako Kakuno, Akira Miyauchi

\begin{abstract}
Hashimoto's thyroiditis is an autoimmune disease that usually requires medical treatment but sometimes benefits from surgical treatment. The reasons why patients with Hashimoto's thyroiditis requires thyroidectomy are (a) malignant lymphoma (b) repeated acute exacerbation of thyroiditis and (c) concern of coexisting thyroid neoplasm from Hashimoto's thyroiditis. We present three patients with Hashimoto's thyroiditis, a 69-year-old woman with MALT lymphoma, a 53-year-old woman with repeated episodes of neckpain and a 67-year-old woman with a coexistent benign thyroid nodule. These three patients with Hashimoto's thyroiditis received surgical treatment and had an excellent outcome and document that some patients with Hashimoto's thyroiditis warrant surgical treatment.
\end{abstract}

Keywords: Thyroid surgery, Autoimmune thyroiditis.

How to cite this article: Kikuchi S, Matsuzuka F, Aihara T, Yasui C, Yanagi H, Mitsunobu M, Yamanaka N, Kakuno A, Miyauchi A. What should Autoimmune Thyroiditis be considered for? World J Endoc Surg 2012;4(2):54-59.

Source of support: Nil

Conflict of interest: None declared

\section{INTRODUCTION}

Hashimoto's thyroiditis is an autoimmune disease. Patients with Hashimoto's thyroiditis are predisposed to the development of thyroid lymphoma, may have local and systemic symptoms or coexisting benign and malignant thyroid disorders. Indications for thyroidectomy in patients with Hashimoto's thyroiditis are limited to the development of lymphoma, severe compression symptoms, enlargement of thyroid struma and repeated episodes of pain. ${ }^{1-14}$ Most studies have indicated that indications for operation are excessive enlargement of the thyroid (>100 gm), severe symptoms (compression, dysphasia) and malignancy. ${ }^{1-14}$ Overall thyroidectomy is only necessary in a small number of patients with Hashimoto diseases, but in some cases, surgical intervention is necessary and helpful. We report three cases of the patients with Hashimoto's thyroiditis who received surgical treatment and had an excellent outcome.

\section{CASE REPORTS}

\section{Case 1}

A 69-year-old woman with hypothyroidism received thyroid hormone for 4 years prior to her thyroidectomy. Her thyroid goiter was goitrous and quite large.
Preoperative laboratory tests showed that thyroid stimulating hormone (TSH) was $0.15 \mu \mathrm{IU} / \mathrm{ml}$, free thyroxine (FT4); $1.43 \mathrm{ng} / \mathrm{ml}$, free triiodothyronine (FT3); $3.22 \mathrm{pg} / \mathrm{ml}$, antithyroid peroxidase (anti-TPO) antibody; $112 \mathrm{U} / \mathrm{ml}$ and anti-thyroglobulin (anti-Tg) antibody; $0.3 \mathrm{U} / \mathrm{ml}$. Findings of ultrasonography revealed an enlarged heterogeneous hypoechoic lesion.

Fine needle aspiration cytology (FNAC) suggested lymphoma (Figs 1A to D). Total thyroidectomy was performed. A weight of specimen was $102 \mathrm{gm}$. Pathological finding diagnosed mucosa-associated lymphoid tissue (MALT) lymphoma showing small B cells with a lymphoid epithelial lesion (LEL) (Fig. 2). Laboratory testing revealed immunoglobulin $\mathrm{H}$ rearrangement (Figs $3 \mathrm{~A}$ and $\mathrm{B}$ ).

Postoperatively, the patient developed transient hoarseness but no tetany. Radiation treatment of 40 Gy was administered to her thyroid bed. There has been no recurrence during the two years of follow-up treatment.

\section{Case 2}

A 53-year-old woman who had taken thyroid hormone supplements for Hashimoto disease and had also taken NSAIDS because of worsening and anterior severe neck pain. Physical examination revealed an enlarged, firm thyroid of size $8 \times 6 \mathrm{~cm}$.

Findings of ultrasonography showed a hypoechoic lesion in whole thyroid.

Preoperative investigations revealed that her TSH was $0.006 \mu \mathrm{IU} / \mathrm{ml}, \mathrm{FT} 4 ; 1.46 \mathrm{ng} / \mathrm{ml}$, FT3; $2.83 \mathrm{pg} / \mathrm{ml}$, anti-TPO antibody; $>600 \mathrm{U} / \mathrm{ml}$ and anti-TG antibody; $>400 \mathrm{U} / \mathrm{ml}$. A total thyroidectomy was performed because of her persistent symptoms and an 80 gm goiter was removed. Both recurrent nerves and one parathyroid gland on the left side and one on the right side were identified and preserved, while the right lower parathyroid was autotransplanted. Pathological diagnosis was a Hashimoto disease. Postoperatively, the patient developed transient hypoparathyroidism, which improved after treatment with calcium and vitamin supplementation for 6 months (Figs 4A to D).

\section{Case 3}

A 67-year-old woman with known Hashimoto disease was admitted with a right thyroid tumor, measuring $1.2 \times 1.0 \mathrm{~cm}$. Preoperative tests showed that TSH was $1.769 \mu \mathrm{IU} / \mathrm{ml}$, FT4; $0.80 \mathrm{ng} / \mathrm{ml}$, FT3; $3.02 \mathrm{pg} / \mathrm{ml}$ and anti-Tg antibody; $13.6 \mathrm{U} / \mathrm{ml}$. 
Ultrasonography findings revealed a hypoechoic tumor with vascular supply. FNAC findings suspected a follicular neoplasm (Figs 5A and B). A right thyroidectomy was performed and histology revealed a Hashimoto disease with tumor formation (Figs 6A to C).

\section{DISCUSSION}

Thyroid cancer concomitant with Hashimoto's thyroiditis requires surgical treatment, and the decision for thyroidectomy is usually based on cytological findings after FNA biopsy. The treatment of thyroid lymphoma is usually with chemotherapy or radiation therapy or both but thyroidectomy is also effective. ${ }^{12}$ Matsuzuka et al reported that among 119 cases of thyroid lymphomas, 40\% cases were hypothyroid and Hashimoto's thyroiditis is the risk factor for thyroid lymphomas. ${ }^{12}$ Total thyroidectomy or lobectomy has been recommended for thyroid lymphomas. ${ }^{12}$

Patients with Hashimoto's thyroiditis without discrete symptoms or nodules should be followed with an annual sonography to rule out thyroid lymphoma or coexistent tumors. $^{13}$

Rapid enlargement of the tumor, dysphasia and compression suggest thyroid lymphoma. Clinically, it is sometimes difficult to differentiate between Hashimoto's
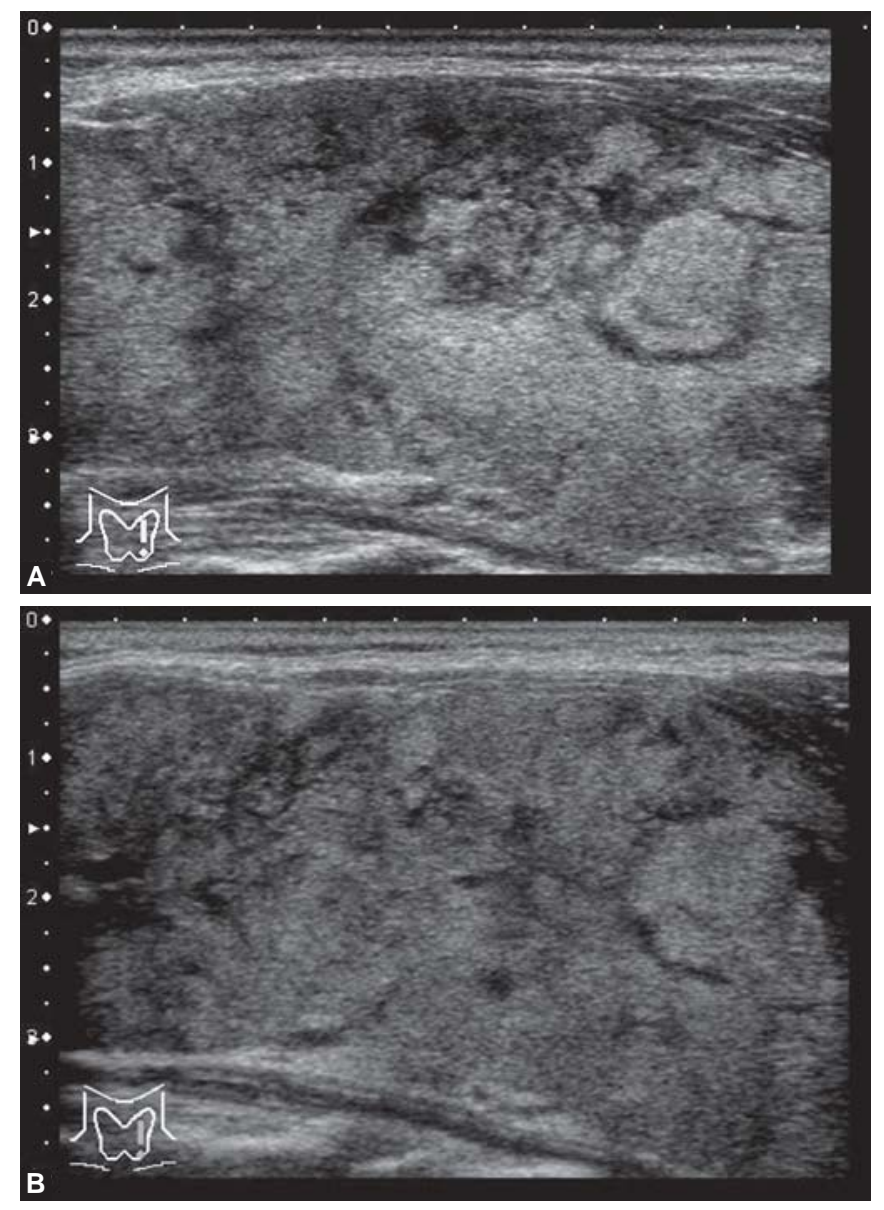

thyroiditis and thyroiditis with coexisting lymphoma. The identification and detection of enlargement of hypoechoic lesions by ultrasonography are strongly indicative of lymphomas; FNAC, needle biopsy or open biopsy should be performed in such cases. After histological examination, the lymphoma (B-cell type or T-cell type) can be typed by

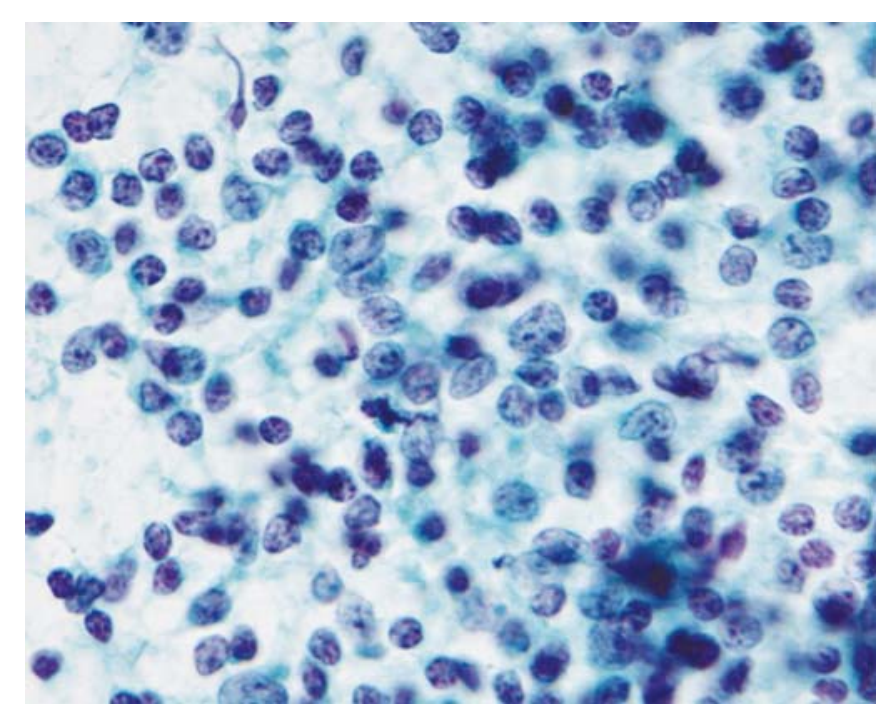

Fig. 2: Fine needle aspiration cytology findings: Suspected lymphoma
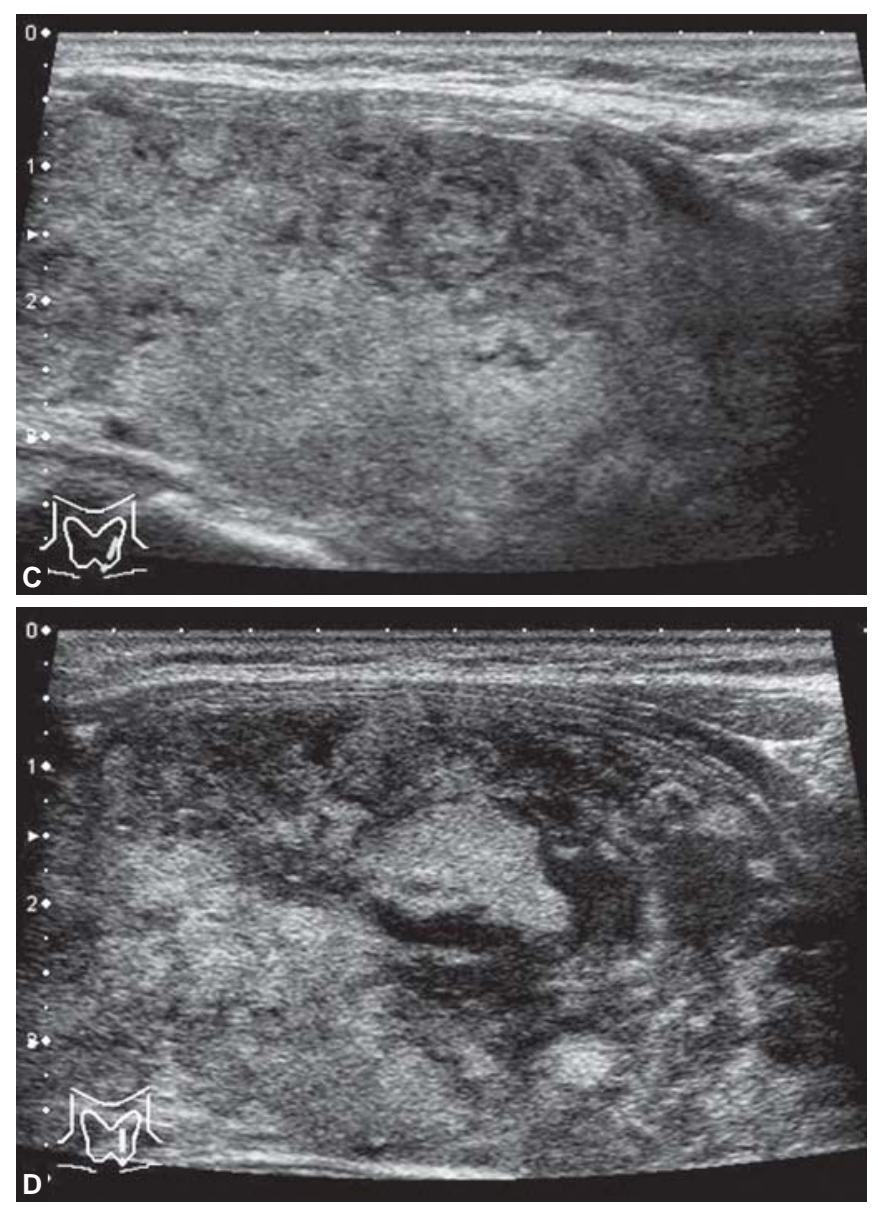

Figs 1A to D: Findings of ultrasonography: Enlarging hypoechoic lesion; (A) first examination, (B) one-year follow-up, (C) one-year and 6-month follow-up, (D) two-year follow-up 
$\mathrm{HE}, \mathrm{K}$ and $\gamma$ staining and analysis of immunoglobulin gene rearrangement. ${ }^{14}$

Most thyroid lymphomas, 2 to 5\% of thyroid malignancies but less than $2 \%$ of extranodular lymphomas are non-Hodgkin B cell type. ${ }^{12}$ Most thyroid lymphomas occur in patients with Hashimoto's thyroiditis. ${ }^{14}$ The risk of lymphoma in patients with Hashimoto thyroiditis is 70 to 80 times higher than that in patients without Hashimoto's thyroiditis. ${ }^{14}$ Furthermore, most patients with thyroid lymphomas are older women (>70 years) with
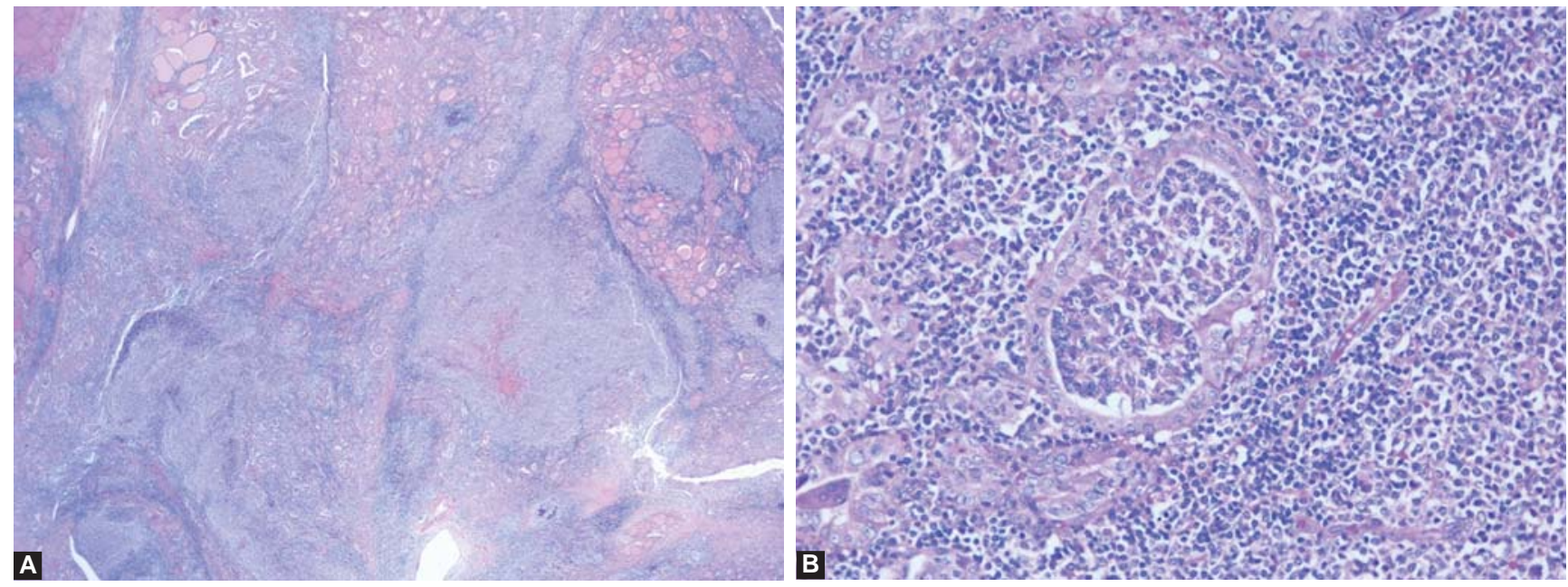

Figs 3A and B: Pathological diagnosis: Mucosa-associated lymphoid tissue (MALT) lymphoma showing small B cells with a lymphoid epithelial lesion (LEL): (A) HE x 100, (B) HE x 200
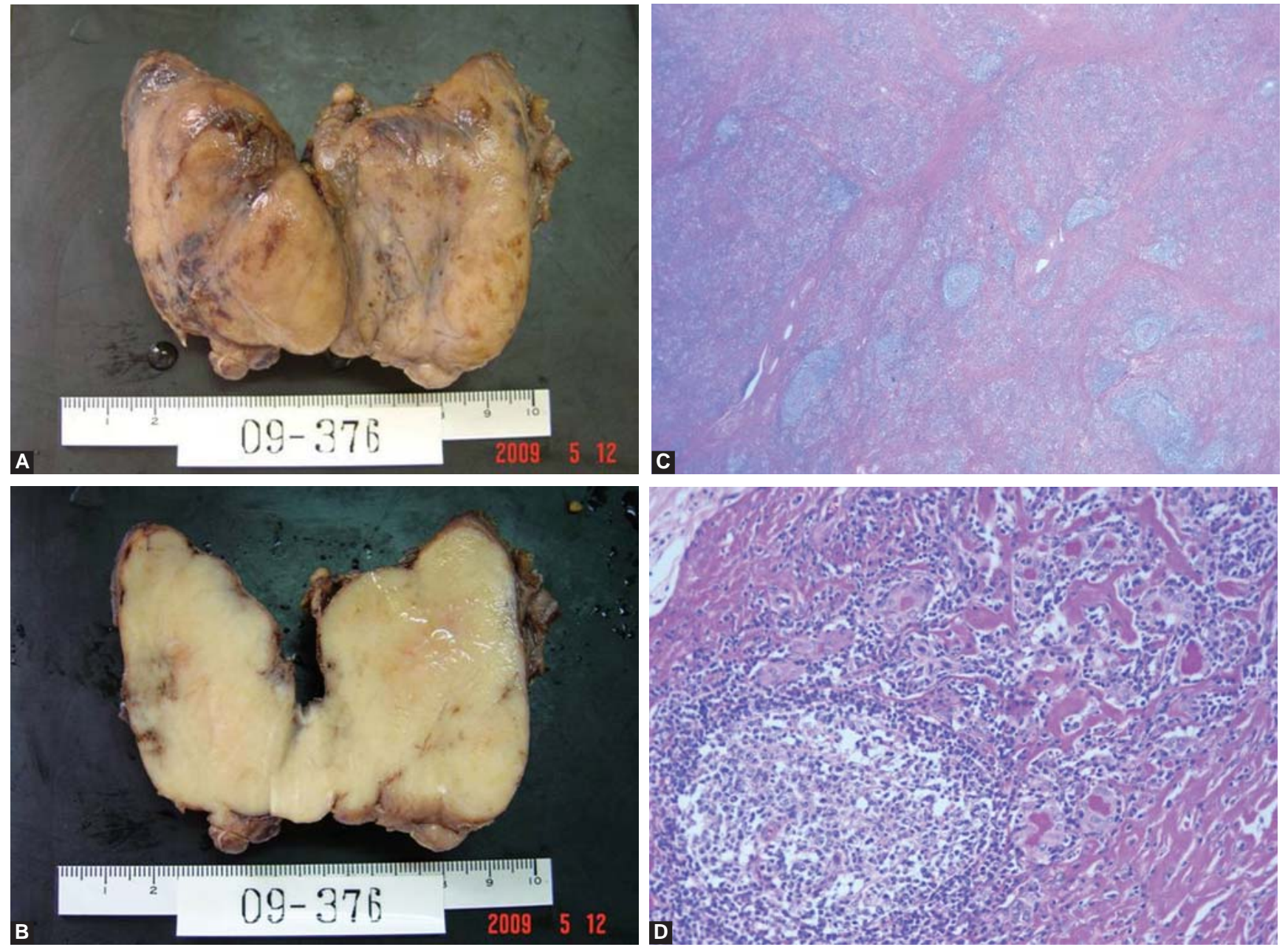

Figs 4A to D: Pathological diagnosis: Hashimoto disease. The lymphoid tissue is distributed within and around the lobules and it invariably exhibits large follicles with prominent germinal centers. (A) Whole aspect of the specimen, (B) cut surface of the specimen, (C) HE x 100, (D) HE x 200 
Hashimoto's thyroiditis. Most of these patients with Hashimoto's thyroiditis are asymptomatic, but sometimes have hoarseness, dysphasia and hypothyroidism. Thyroid function is normal in 58\% of patients. ${ }^{13}$ Findings on physical examination reveal a firm, hard moderately enlarged goiter.
There are two types of lymphoma, MALT and large cell types. Detection of enlargement of a hypoechoic lesion on ultrasonography suggests MALT lymphoma. Patients with MALT lymphomas usually have few signs of rapid enlargement. MALT lymphoma without rapid enlargement is difficult to differentiate from Hashimoto's thyroiditis on
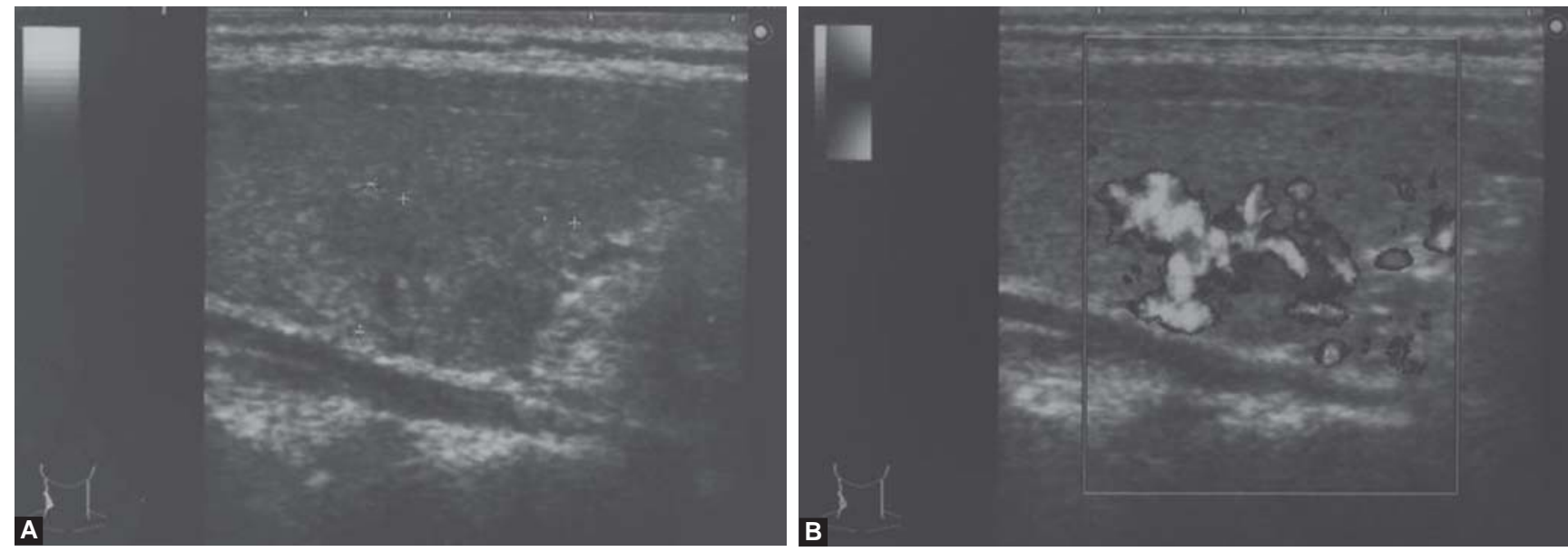

Figs 5A and B: Ultrasonography findings: Hypoechoic tumor (A), with vascular supply (B)
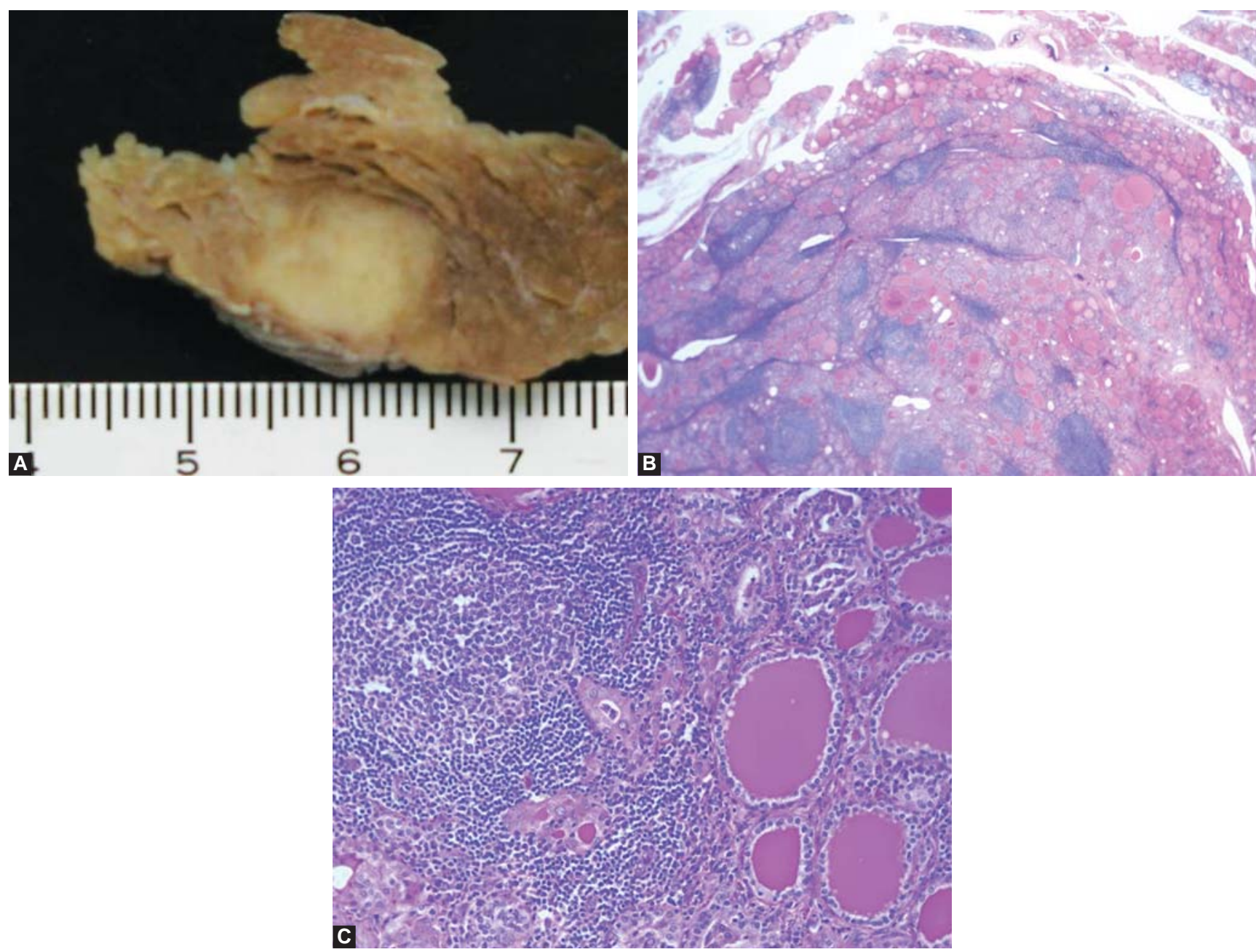

Figs 6A to C: Pathological diagnosis: Hashimoto disease with tumor formation. The thyroid follicles are small and atrophic and lymphocytic infiltration of stroma is seen. The lymphoid tissue exhibits large follicles with prominent germinal centers. (A) Cut surface of the specimen, (B) HE x 100, (C) HE x 200 
the basis of histologic findings. Thyroid lobectomy or total thyroidectomy, instead of open biopsy, is recommended for establishing the final diagnosis. Most thyroid MALT lymphomas are in the clinical stage I E and are treated by total thyroidectomy and external radiation therapy only. ${ }^{3,4}$ On the contrary, core needle or open biopsy is usually sufficient to diagnose large cell lymphomas because they usually grow rapidly and show clear signs of malignancy. Treatment options for large cell lymphomas are radiation and chemotherapy (CHOP therapy). ${ }^{4,5}$

Our patient was monitored by periodical ultrasonography and FNAC was performed because the hypoechoic lesions showed gradual enlargement. When the diagnosis of MALT lymphoma is delayed, it can progress to large cell lymphoma, which has a poor prognosis. Luckily, the diagnosis in our case was established at an early stage because the patient had been monitored with periodical ultrasonographic examinations.

Acute exacerbation of Hashimoto's thyroiditis differs from those of subacute thyroiditis and has different histological characteristics and associates laboratory abnormalities. It is sometimes difficult to differentiate these two disease by clinical findings alone. Antithyroid antibody testing helps to make this diagnosis. When the antibody is negative, subacute thyroiditis or diffuse goiter is considered, and when it is positive, acute exacerbation of Hashimoto's thyroiditis is suggested. Acute exacerbation of Hashimoto's thyroiditis is usually treated by steroids, but sometimes the symptoms worsen on discontinuing steroids or patients relapse. ${ }^{6,7}$ Thyroidectomy is useful in patients wherein discontinuation of steroid treatment is contraindicated and in patient with recurrence disease. ${ }^{7}$

Nontender Hashimoto's thyroiditis rarely leads to systemic inflammation, but some patients have persistent fever and benefit from treatment with thyroid surgery. ${ }^{6-8}$ Histopathological findings include extensive fibrosis and destruction of the thyroid anatomy. ${ }^{7}$ Kubota et al suggested that inflammation disappearing by thyroid surgery indicates that the inflammatory cytokine production originated only from the thyroid; they also elucidated that by positive interleukin (IL) $1 \alpha$, IL1 $\beta$ and tumor necrosis factor (TNF) $\alpha$ staining. ${ }^{8}$ Our case is similar to the cases reported in the past. ${ }^{6,7,9,10}$ We performed total thyroidectomy and treated postoperative temporary hypocalcemia with calcium and vitamin D supplementation. Thyroid functions were normal with thyroid hormone supplementation. She has no clinical recurrent episodes one year following thyroidectomy.

We recommend total thyroidectomy for patients with recurrent, symptomatic thyroiditis which is a relatively uncommon.

One case of tumor formation in Hashimoto's thyroiditis was reported in Japan. ${ }^{11}$ We recommended surgery for our patient because the FNAC findings were suggestive of follicular neoplasm. However, histopathology only revealed Hashimoto's thyroiditis. Tumor formation in Hashimoto's thyroiditis is difficult to diagnose by FNAC. ${ }^{11}$

In our case, we performed FNAC once, which showed that the resumed tumor was follicular in nature. FNAC could have been repeated in our case before undertaking surgery. However, we performed surgery because ultrasonography showed an irregular, hypoechoic lesion and cytology was a follicular neoplasm. We think that this case of tumor formation in Hashimoto's thyroiditis should be carefully followed up.

\section{SUMMARY}

We present three cases of surgical intervention in patients with Hashimoto's thyroiditis complicated by MALT lymphoma, repeated acute exacerbations of symptomatic thyroiditis and coexisting tumor formation.

Although Hashimoto disease is an autoimmune disorder, surgical intervention may be required in some cases.

\section{REFERENCES}

1. Samoan NA, Ordonez NG. Uncommon types of thyroid cancer. Endocrinol Metab Clin North Am 1990;19:637.

2. Holm LE, Blomgren H, Lowhagen T. Cancer risks in patients with chronic lymphocytic thyroiditis. N Eng J Med 1985; 312:601.

3. Skarsgard ED, Connors JM, Robins RE. A current analysis of primary lymphoma of the thyroid. Arch Surg 1991; 126:1199.

4. Pyke CM, Grant CS, Habermann TM. Non-Hodgkin’s lymphoma of the thyroid: Is more than biopsy necessary? World J Surg 1992;16:604.

5. Kanachi A. Diagnosis for thyroid lymphoma. Jibiinkoka Risho (Jpn J clinical ENT) 1992;85:1301.

6. Kong YC, DeGroot LJ. Painful Hashimoto`s thyroiditis as an indication for thyroidectomy: Clinical characteristics and outcome in seven patients. J Clin Endocrinol Metab 2003;88: 2667.

7. Ohye H, Fukata S, Kubota S, Sasaki I, Takamura Y, Matsuzuka F, et al. Successful treatment for recurrent painful Hashimoto's thyroiditis by total thyroidectomy. Thyroid 2005; 15:340.

8. Kubota S, Matsuzuka F, Ohye H, Nishihara E, Kudo T, Ito M, et al. Sustained fever resolved promptly after total thyroidectomy due to huge Hashimoto's fibrous thyroiditis. Endocr 2007;31: 88-91.

9. Ishihara T, Mori T, Waseda N, Ikekubo K, Akamizu T, Imura H. Pathological characteristics of acute exacerbation of Hashimoto's thyroiditis — serial change in a patient with repeated episodes. Endocrinol Jpn 1986;33:701.

10. Ishihara $\mathrm{T}$, Mori $\mathrm{T}$, Waseda $\mathrm{N}$, Ikekubo $\mathrm{K}$, Akamizu $\mathrm{T}$, Imura $\mathrm{H}$. Histological, clinical and laboratory findings of acute exacerbration of Hashimoto's thyroiditis - comparison with those of subacute granulomatous thyroiditis. Endocrinol Jpn 1987;34:831. 
11. Solivetti FM, Bacaro D, Cecconi P, Baldelli R, Marandino F. Small hyperechogenic nodules in thyroiditis: Usefulness of cytological. J Exp Clin Cancer Res 2004;23:433.

12. Matsuzuka F, Miyauchi A. Clinical aspects of primary lymphoma: Diagnosis and treatment based on our experience of 119 cases. Thyroid 1993;3:93.

13. Kato I, Tajima K, Suchi T, Aozasa K, Matsuzuka F, Kuma K, et al. Chronic thyroiditis as a risk factor of B-cell lymphoma in the thyroid gland. Jpn J cancer Res 1985;76:1085.

14. Matsuzuka F, Fukata S, Kuma K, Miyauchi A, Kakudo K, Sugawara M. Gene rearrangement of immunoglobulin as a marker of thyroid lymphoma. World J Surg 1998;22:558.

\section{ABOUT THE AUTHORS}

\section{Shoichi Kikuchi (Corresponding Author)}

Chief, Department of Surgery, JSDF Kumamoto Hospital Kumamoto, Japan, Phone: +81-96-368-5111, Fax: + 81-96-368-2720 e-mail: shoichi_kikuchi@hotmail.com

\section{Fumio Matsuzuka}

Chief, Department of Surgery, Meiwa Hospital, Hyogo, Japan

\section{Tsukasa Aihara}

Chief, Department of Surgery, Meiwa Hospital, Hyogo, Japan

\section{Chiaki Yasui}

Chief, Department of Surgery, Meiwa Hospital, Hyogo, Japan

\section{Hidenori Yanagi}

Chief, Department of Surgery, Meiwa Hospital, Hyogo, Japan

\section{Masao Mitsunobu}

Chief, Department of Surgery, Meiwa Hospital, Hyogo, Japan

\section{Naoki Yamanaka}

Director, Department of Surgery, Meiwa Hospital, Hyogo, Japan

\section{Ayako Kakuno}

Chief, Department of Pathology, Meiwa Hospital, Hyogo, Japan

\section{Akira Miyauchi}

Director, Department of Surgery, Kuma Hospital, Hyogo, Japan 